\title{
Joint Observation Planning and Follow-ups
}

\author{
Gemma Anderson ${ }^{1}$ \\ International Centre for Radio Astronomy Research, Curtin University \\ GPO Box U1987, Perth, WA 6845, Australia \\ E-mail: gemma.andersonecurtin.edu.au
}

\section{Eric Chassande-Mottin}

APC, AstroParticule et Cosmologie, Université Paris Diderot, CNRS/IN2P3, CEA/Irfu, Observatoire de Paris, Sorbonne Paris Cité, F-75205 Paris Cedex 13, France

E-mail: ecm@apc.in2p3.fr

The aim of this workshop was to discuss how to implement more flexible operating models for joint or target of opportunity observations of multi-messenger events. While several obstacles exist that prevent far reaching standardisation of software and infrastructure for facilitating such coordination, several recommendations were made that could improve the opportunities for joint observations and follow-ups in the future. These include the idea that observatories, facilities and scientists need to agree upon what data can be shared and to also publicise their observing schedules, there is a continuation of the standardisation of communication protocols for transient and multi-messenger events, that new software implementations for coordinating joint observing between facilities be open source, and that the multi-messenger community become actively involved in the making of observatory transient policy.

The New Era of Multi-Messenger Astrophysics - Asterics2019

25 - 29 March, 2019

Groningen, The Netherlands

\section{${ }^{1}$ Speaker}




\section{Introduction}

As we enter the era of multi-messenger astrophysics, our desire to perform multi-messenger follow-up of astronomical sources and transient events is motivated by the fact that the knowledge gained is so much more than the sum of its parts. The follow-up campaigns for recent, exciting multi-messenger events (e.g. the V404 Cyg black hole outburst, the binary neutron star (BNS) merger GW170817, the neutrino-detected blazar TXS 0506+056, and GRB 190114C detected by the Major Atmospheric Gamma Imaging Cherenkov Telescope [MAGIC]) took place rather organically with groups of astronomers scheduling observations autonomously, and independently of others' decisions. We discuss the question as to whether coordinated and organised observations are possible with the hope to achieve more robust results, and with more efficiency. To do so, we would therefore need to implement more flexible operating modes for joint and/or target of opportunity observations to ensure we can draw upon a wide range of resources, instruments and technology that is necessary for archiving the best scientific return.

Joint observations mean either coincident/simultaneous observations of the same source or coordination/triggered observations of the same source that are not necessarily simultaneous. The vision for multi-messenger astrophysics is to be able to coordinate, automate and standardise our strategies, while moving towards a more open skies and open software community.

One example of a program working towards a unified and real-time analysis of incoming data-streams from high-energy and multi-messenger facilities is the Astrophysical Multimessenger Observatory Network (AMON; [1]). AMON searches for subthreshold events correlated in time and space, and generates significant transient alerts. There is also a network of participating follow-up facilities that provide rapid (and in many case automated) multiwavelength and multi-messenger observations of AMON events. AMON is therefore an excellent example of a joint observing program; but AMON currently does not allow coordinated observation scheduling. In the following proceedings, we discuss some of the strategies for joint observing planning and follow-up presented during this conference (Section 2), followed by recommendations on improvements to better facilitate this coordination in the future (Section 3).

\section{Presented Joint Analysis strategies}

Following the conference presentations, three key strategies became evident for the joint analysis and follow-up of multi-messenger events. The first is the automation of the rapid followup of transient events. The second was the presentation of relevant software that are specifically designed to standardise observation scheduling or provide toolkits to coordinate observations with multiple observatories world-wide. The third strategy is automated temporal and positional correlation of transient alert data-streams. In the following, we outline the active initiatives.

\subsection{Rapid-response/automated transient triggering}

VOEvents are the standard for broadcasting alerts related to transient events set out by the International Virtual Observatory Alliance (IVOA; [2]). Many multi-messenger facilities disseminate VOEvents of newly discovered transients including the Swift Burst Alert Telescope (BAT), the Fermi Gamma-ray Burst Monitor (GBM), Advanced LIGO and Virgo (aLIGO/Virgo), the Antares and IceCube Neutrino Observatories, and the All-Sky Automated Survey for Supernovae (ASASSN), just to name a few. Several software packages have been written to 
enable the parsing and filtering of VOEvents that can then allow for the direct automation of transient follow-up. The most utilised of these software packages include the '4 Pi Sky VOEvent' broker [3] and the Comet VOEvent client [4], which are used by facilities such as the Murchison Widefield Array (MWA), the Low-Frequency Array (LOFAR), the Australia Telescope Compact Array (ATCA), Antares, IceCube, the High Energy Stereoscopic System (H.E.S.S.), the Zwicky Transient Facility (ZTF), along with future plans for the Cherenkov Telescope Array (CTA) and the next generation neutrino telescope KM3Net. This is an excellent demonstration of how a useful and well documented tool can become widely used by the community.

\subsection{Combined/joint telescope programs and multi-observatory coordination}

There are already several joint observing programs that coordinate observations between observatories. For example, XMM-Newton has joint observing programs with 9 different observatories across the electromagnetic spectrum, with the inclusion of two multi-messenger facilities ${ }^{2}$. There are also coordinated observing opportunities between the Chandra X-ray Observatory and the radio facilities run by the National Radio Astronomy Observatory (NRAO) ${ }^{3}$. During the conference, several programs were presented that aim to standardise scheduling strategies and resource optimisation for joint observing programs. For example, the Astronomical Event Observatory Network $(\mathrm{AEON})^{4}$ led by Las Cumbres Observatory coordinates a fleet of world-class telescopes with highly flexible queue scheduling for the rapid follow-up and characterisation of transients, which will be particularly important in the Large Synoptic Survey Telescope era. The observing requests, schedule coordination and data sharing for AEON are standardised by the Target and Observation Manager (TOM) Toolkit. ${ }^{5}$ Other programs include the Global Relay of Observatories Watch Transients Happen (GROWTH) Marshall led by Caltech ${ }^{6}$, which ensures the potential for $24 \mathrm{hr}$ monitoring of the transient sky that is "unbeaten by sunrise". Further IVOA standards for determining instrument source visibility (ObsVisSAP) and generating observing schedules (ObsLocTAP) are also being designed and implemented as standards for high-energy telescopes, including Chandra, XMM, Swift and Integral.

\subsection{Automated temporal and positional correlation}

There are several programs set to automate the temporal and positional correlation of transient alert streams. The correlation of subthreshold multi-messenger events via AMON has already been described in Section 1. Another example includes the Alert Management, Photometry and Evaluation of Lightcurves (AMPEL) transient processing and analysis package [5], which is currently run on IceCube neutrino data with the transient alert stream generated by ZTF, with the aim of identifying multimessenger matches. AMPEL offers the capability of contributing additional transient input channels for correlation with the ZTF alert streams.

\footnotetext{
${ }^{2} \mathrm{https}$ ://xmm-tools.cosmos.esa.int/external/xmm user support/documentation/AOpolicy/

${ }^{3} \mathrm{https} / / /$ science.nrao.edu/observing/call-for-proposals/2019b/chandra

${ }^{4}$ https://lco.global/aeon/

${ }^{5} \mathrm{https://1co.global/tomtoolkit/}$

${ }^{6}$ http://growth.caltech.edu/
} 


\section{Discussion}

To make joint observations a reality, it is important to collect more use cases and document our experience of gathering multi-wavelength and multi-messenger observations of exciting events. As the sample grows, we can start to look for those areas requiring further infrastructure development. Based on the summary in Section 2, it is clear several work-flows, software and coordinated observing programs are currently being designed and implemented to facilitate joint observations and follow-up of multimessenger events.

The discussion in this workshop session was aimed at identifying strategies to better facilitate and coordinate joint observations of future multimessenger events. As part of this discussion, several general obstacles were identified. These include the fact that transient followup requires target-of-opportunity ( $\mathrm{ToO}$ ) observations that are competing with more standard programs, and this can create tension between observers. In addition, multi-messenger astrophysics is quickly becoming recognised as a high impact field so competition also becomes an obstacle to coordination and transparency. In the context of this discussion, it is important to realise that every telescope, experiment and project have different priorities, and trying to coordinate more outreaching initiatives requires funding and a "buy-in" from participating facilities. In the following, we make several high-level suggestions on how the multimessenger community could move toward a more joint observation and follow-up strategy.

\subsection{Observatories, facilities and scientists need to agree upon what data can be shared.}

This is an initial zero-cost step towards joint and coordinated observations. Many visualisation clients are already providing a great incentive to share their data and catalogues (e.g. TOPCAT and the Aladin Sky Atlas; [6], [7], [8]). It is important to note that data sharing needs to rely on mature and well-documented analysis tools that are widely accessible and understandable.

\subsection{Telescope schedules should be public}

There are many telescopes that perform frequent all-sky surveys, with much of the resulting data becoming instantly available online (e.g. Swift). Publicising schedules ensure that other facilities can manually or automatically schedule simultaneous observations of their favourite source. Knowledge of 'big' telescope schedules can therefore open up opportunities for smaller telescopes. However, it can be difficult to define or obtain reliable schedules for a given night as they can change at the last minute (e.g. Swift, Chandra), or contain proprietary information.

One way to make schedule sharing a reality is to create virtual observatory (VO) standards that allow for a standardised sharing of this information, which is already being implemented by $\mathrm{X}$-ray observatories (see Section 2.2). However, in most cases observing schedules are constructed by a team of astronomers rather than by software. It would therefore be useful for such schedule teams to be more proactive and look for opportunities to automate and share.

\subsection{Standardisation of communication protocols for transient and multi-messenger events}

The standardisation of transient message format is essential. This has been well facilitated by the IVAO VOEvents and many facilities make use of software designed to interface with VOEvent streams (see Section 2.1). While communication protocols should be standardised, enforcing a triggering software standard across all triggering facilities is not realistic given the diversity in software and hardware platforms, along with their different operational modes and science priorities. In addition, such software portability would require large efforts to implement and potentially prevent the evolution of individual facility platforms. 


\subsection{New software implementations to coordinate joint observing needs to be open source}

Much of the software development described in Section 2 could potentially seed larger infrastructure. However, the standardisation of the tools may not come from small groups, but rather be driven by institutions or funding agencies. By making these software platforms open source, it makes it easier for teams/observatories/facilities to participate in joint observations and also dissemination relevant information regarding observations and data access.

\subsection{Provide suggestions for observatory transient policies}

For many facilities, if observing time is lost due to a ToO observation, that time is not necessarily recoverable later in the semester, nor considered for the next round of observing proposals. Many telescopes also change instruments or configurations, which means that lost time can be devastating if observers need to wait an entire year to finish their programmes.

As we move into the multi-messenger era, and more time-critical events are detected and require follow-up, observatories need to be encouraged to provide compensation to interrupted programmes to prevent an escalation in tension between groups. Many radio telescopes have alternative and flexible policies to deal with ToO overrides. One suggestion is for observatories to schedule unassigned 'make-up' time at the end of the observing semester/instrument/configuration that can be allocated to interrupted programs (e.g. ATCA).

The Time Allocation Committees also have an important policy role to play when evaluating triggering and ToO proposals. For example, both ATCA and MWA rely on proposal rankings to prioritise programs, and request overrideability statements. Such statements are particularly important when the instrument has automated triggering capabilities, where a delay in the requested time-critical observation would prevent the science goal being reached. Other facilities (e.g. LOFAR) sets a maximum acceptable number of triggers for a triggering program.

Another complication is that transient teams tend to 'save up' their transient overrides. This results in an accumulation of ToO usage at the end of the observing era in order use time allocations and ensure similar allocations in the future. There are other complications related to multimessenger programs (e.g. aLIGO/Virgo O3 run) being misaligned with observing semesters. One option is to allocate longer observing programs for transient science. For example, the European Southern Observatory (ESO) allowed ToO programs for their instruments of $>1.5 \mathrm{yrs}$ for gravitational wave follow-up, rather than the standard 6 month proposal renewal. Of course, telescope oversubscription is still a problem so there is an argument for more dedicated telescopes like ZTF, which does transient optical surveys, the transient spectrograph Son of X-Shooter (SoXS) on the ESO New Technology Telescope, and the Arcminute Microkelvin Imager (AMI) radio telescope.

\section{References}

[1]M. W. E. Smith, et al., 2013, The Astrophysical Multimessenger Observatory Network (AMON), Astroparticle Physics, 45, 56

[2]R. Seaman, et al., 2011, IVOA Recommendations: Sky Event Reporting Metadata Version 2.0, arXiv: 1110.0523

[3]T. D. Staley \& R. Fender, 2016, The 4 Pi Sky Transient Alerts Hub, arXiv:1606.03735

[4]J. J. Swinbank, 2014, Comet: A VOEvent broker, Astronomy and Computing, 7, 12

[5]J. Nordin, et al., 2019, Transient processing and analysis using AMPEL: Alert Management, Photometry and Evaluation of Lightcurves, arXiv: 1904.05922

[6]M. B. Taylor, 2005, TOPCAT \& STIL: Starlink table/VOTable Processing Software, ASPC, 347, 29

[7]F. Bonnarel, et al., 2000, The ALADIN interactive sky atlas. A reference tool for identification of astronomical sources, $A \& A S, 143,33$

[8]T. Boch \& P. Fernique, 2014, Aladin Lite: Embed your Sky in the Browser, ASPC, 485, 277 